\title{
COMPLICAÇÕES DECORRENTES DA TERAPIA INTRAVENOSA EM PACIENTES CIRÚRGICOS
}

\author{
Renata Cristina de Campos Pereira*
}

Maria Lúcia Zanetti**

\begin{abstract}
PEREIRA, R.C.de C.; ZANETTI, M.L. Complicações decorrentes da terapia intravenosa em pacientes cirúrgicos.
\end{abstract}
Rev.latino-am.enfermagem, Ribeirão Preto, v. 8, n. 5, p. 21-27, outubro 2000.

A enfermagem desempenha papel primordial na prevenção e redução das complicações relacionadas ao acesso venoso. O estudo teve como objetivo o levantamento das complicações decorrentes da terapia intravenosa. Os dados de maior expressividade obtidos foram: 77,3\% das punções foram realizadas pelo auxiliar de enfermagem; 68\% não usaram luvas durante o procedimento; $60 \%$ das punções tiveram cuidado de enfermagem insatisfatório; 47\% dos dispositivos permaneceram "in situ" de 24 a 72 horas; dentre as complicações, 20\% relacionaram-se com infiltração, 5,4\% infiltração e hematoma e 5,3\% obstrução. Os dados sugerem a necessidade de um aprimoramento da equipe de enfermagem relacionado a terapia intravenosa.

UNITERMOS: infusões endovenosas/complicações, cuidados de enfermagem

\section{INTRODUÇÃO}

Nos Estados Unidos a estimativa anual de infusões através de dispositivos venosos periféricos, acesso venoso, gira em torno de 150 milhões, os quais são realizadas em 30 milhões de pacientes, conforme BREGENZER et al. (1998). Tal acesso é feito pela inserção de um dispositivo em veia periférica e é realizado, principalmente, através da rede venosa dorsal das mãos e antebraços.

Atualmente existem vários tipos de dispositivo no mercado, para os mais diversos propósitos, variando desde o tamanho até o tipo de material de revestimento. No entanto, o acesso venoso pode proporcionar várias complicações ao paciente, como a obstrução do dispositivo, infiltrações locais, inflamações, infecções, com possível septicemia sendo que a mais freqüente delas é a tromboflebite, segundo os estudos de FULTON (1997) e BREGENZER et al. (1998).

Segundo BRANNEN \& SURETTE (1997) as complicações podem estar relacionadas aos fatores físicos e químicos. Dentre os fatores físicos, deve-se levar em conta as técnicas de inserção, a anatomia do local, tamanho e tipo de dispositivo, número de inserções, cateter in situ por mais de 72 horas, a gravidade da doença e infecções preexistentes. Por outro lado, os fatores considerados químicos incluem a infusão de drogas irritantes (como eritromicina intravenosa) e a concentração da infusão (hiper ou hipotônicas). Estudos realizados na década de setenta, nos mostraram que entre 27 e $70 \%$ dos pacientes que recebiam infusões venosas periféricas desenvolviam tromboflebite, variando conforme o tempo de permanência do cateter in situ.

Neste contexto, a equipe de enfermagem possui um papel primordial na prevenção e na redução das complicações relacionadas ao acesso venoso, pois é dela a responsabilidade pelos procedimentos diários de manutenção dos curativos, visto que ocorre uma quebra na integridade cutânea, sendo a flora local o principal foco de infecções.

No Brasil, após uma ampla revisão na literatura foi detectado que há poucos artigos que abordam essa temática (NASCIMENTO, 1994 e VIEIRA, 1998). Considerando que o número de complicações relacionadas às punções venosas é elevado, MAKI \& RINGER (1991), e o escasso número de pesquisa em âmbito nacional e que complicações relacionadas ao acesso venoso poderiam ocasionar seqüelas permanentes ao paciente e elevar o custo hospitalar elaboramos o presente estudo.

\footnotetext{
* Aluna do quinto semestre de graduação da Escola de Enfermagem de Ribeirão Preto da Universidade de São Paulo, bolsista de Iniciação Científica

** Orientadora do projeto, Professor Doutor do Departamento de Enfermagem Geral e Especializada da Escola de Enfermagem de Ribeirão Preto da Universidade de São Paulo
} 


\section{OBJETIVOS}

Analisar os recursos materiais, o tempo in situ do dispositivo, localização e o grau de cuidado de enfermagem e complicações relacionada a terapia intravenosa.

\section{MATERIAL E MÉTODOS}

Estudo de natureza descritiva segundo POLIT \& HUNGHER (1995), realizado com pacientes cirúrgicos internados no décimo andar, seção A, Clínica Cirúrgica, especialidade Gastrocirurgia e Cirurgia Geral de um Hospital de grande porte do interior paulista.

Amostra: quarenta pacientes submetidos a procedimentos cirúrgicos internados nas enfermarias da clínica cirúrgica 1005, 1011 e 1013 (Gastrocirurgia) e 1009, 1015 e 1017 (Cirurgia Geral), no período de 10 de janeiro a 10 de fevereiro de 1999.

Critérios de Inclusão: foram incluídos no estudo os pacientes que: iniciaram a inserção do dispositivo venoso periférico; capazes de responder questões referentes a sinais e sintomas de complicação venosa; não vieram transferidos de outra seção do referido hospital ou de outras Instituições de Saúde, já submetidos a terapia intravenosa prévia; incluídos apenas pacientes adultos, ou seja, maiores de dezoito anos de idade.

Variáveis do Estudo: variáveis relacionadas a caracterização do paciente (especialidade, faixa etária e patologia de base); variáveis relacionadas ao cuidado de enfermagem (grau de cuidado, dispositivos utilizados, motivos de interrupção, calibre, anti-sepsia da pele, condições rede venosa, local de instalação, tipo de restrição e tipo do medicamento: irritantes, hipo e hipermolares).

O grau de cuidado de enfermagem foi definido como se segue: Grau 0-Cuidado de enfermagem satisfatório: dispositivo com boa fixação e limpeza; e o paciente não referiu dor; Grau 1-Cuidado de enfermagem insatisfatório: dispositivo fixado com perda de asas ou linha intravascular, curativo incompleto; o cateter deslocava-se na veia ao movimentar o braço; paciente referiu que o dispositivo estava perfurando a veia; presença de sangue na parte externa do dispositivo ou no curativo e Grau 2-Cuidado de enfermagem muito insatisfatório: quando o dispositivo era mantido pelo curativo e deslocava-se na veia, paciente referiu que o dispositivo estava perfurando a veia e dispositivo in situ há mais de cinco dias.

Elaboração do Instrumento de Coleta de Dados: um roteiro sistematizado foi construído considerando as variáveis do estudo, a experiência pessoal do pesquisador e dados dos estudos de LUNDGREN et al. (1993) e NASCIMENTO (1994). O instrumento contém dezenove questões fechadas contendo as variáveis relacionadas a caracterização do paciente cirúrgico e às referentes ao cuidado de enfermagem.

\section{Validação do Instrumento}

Foi solicitado a colaboração de três enfermeiras especialistas e para a chefe da Divisão de Enfermagem do hospital em estudo para apreciar o instrumento quanto à clareza dos itens, facilidade de leitura, compreensão e forma de apresentação. $\mathrm{O}$ estudo piloto foi realizado, no período de 14 de dezembro a 17 de dezembro de 1998 , com quatro pacientes.

Coleta de Dados: os dados foram obtidos através da observação direta e pelo próprio pesquisador. Primeiramente obteve-se a escala de cirurgia e assim, com um dia de antecedência certificava-se quais os pacientes que iriam para cirurgia. No dia da cirurgia, o pesquisador comparecia ao local às cinco horas e quarenta e cinco minutos e acompanhava a instalação da terapia intravenosa anotando as respostas no próprio instrumento, concomitantemente, a observação, sem fazer perguntas ao funcionário. Após o término do procedimento e saída do funcionário da enfermaria, perguntava-se ao paciente se gostaria de fazer algum comentário. Assim, o pesquisador permanecia junto ao paciente, de cinco a trinta minutos, período este que antecedia o chamado para o centro cirúrgico. Após esta etapa realizava a observação de outros pacientes que haviam iniciado e mantinham a terapia intravenosa. Todos os dias a prescrição médica era verificada com o objetivo de certificar-se quais os pacientes que teriam a terapia interrompida, a fim de acompanhar o procedimento de suspensão.

Quando o pesquisador verificava que a punção encontrava-se em outro local que não o da punção original para cirurgia, obteve-se através do paciente, os dados quanto o motivo, horário e local da interrupção. A observação ocorreu das cinco horas e quarenta e cinco minutos às onze horas e trinta minutos no período da manhã. Às quinze horas, o pesquisador retornava a seção e aguardava a chegada dos pacientes do centro de recuperação anestésica e continuava a observação até às dezoito horas.

Organização dos Dados para Análise: aos dados contidos no formulário foram atribuídos códigos específicos para cada variável selecionada e transpostas para uma planilha. A estrutura do banco de dados foi confeccionada a partir das planilhas sendo formatado no programa Word (para Windows 95 versão 6.0). A análise dos dados obedeceu às normas para um estudo descritivo.

Aspectos Éticos: o estudo foi realizado após a aprovação do Comitê de Ética em Pesquisa do hospital em estudo, em 07 de janeiro de 1999. O pesquisador esclareceu os sujeitos quanto aos objetivos e natureza da 
investigação. A observação iniciou-se mediante a assinatura do termo de consentimento esclarecido pelos pacientes.

\section{RESULTADOS E DISCUSSÃO}

Apresentaremos os resultados e a discussão de acordo com a seqüência dos objetivos propostos para a presente investigação.

Dos 40 pacientes submetidos a punção venosa internados em um hospital de grande porte, $24(60 \%)$ estavam internados em enfermarias de cirurgia geral e $16(40 \%)$ gastrocirurgia. Em relação a faixa etária, observamos que $50 \%$ dos pacientes internados encontram-se entre 48 a 78 anos de idade.

LUNDGREN et al. (1993) ao analisar o cuidado de enfermagem em pacientes clínicos e cirúrgicos com presença de dispositivos intravenosos obteve que os pacientes cirúrgicos eram mais idosos que os clínicos.
Em relação a faixa etária, observamos que a maioria dos pacientes cirúrgicos internados submetidos a terapia intravenosa encontra-se na faixa etária 58 a 68 anos $(22,5 \%)$ e 68 a 78 anos $(20 \%)$ respectivamente. NORWOOD et al. (1991) referem que a idade constitui um dos fatores de risco predisponentes a infecção relacionada ao cateter venoso central e cateter venoso periférico, pois altera os mecanismos de defesa orgânica do indivíduo. Considerando que os pacientes investigados encontram-se em sua maioria com idade superior a 48 anos de idade, este dado, isoladamente, já é um fator relevante de risco no planejamento do cuidado de enfermagem em relação a terapia endovenosa a esta clientela.

Quanto a patologia de base, encontramos que $55 \%$ pertenciam a especialidade de gastrocirurgia, sendo que, destas 32,5\% relacionam-se a: colecistopatia crônica calculosa, coledocolitíase, litíase biliar e pólipo vesículo biliar. E que $45 \%$ pertenciam a especialidade cirurgia geral, $37,5 \%$ relacionam-se a hérnias incisionais e inguinais.

Tabela 1 - Distribuição de freqüência de punções venosas realizadas em pacientes internados em um hospital de grande porte, segundo a qualificação profissional da equipe de enfermagem, Ribeirão Preto - SP, 1999

\begin{tabular}{ccccccccc}
\hline Punção & \multicolumn{2}{c}{ Enfermeiro } & \multicolumn{2}{c}{ Auxiliar de enfermagem } & \multicolumn{2}{c}{ Não identificado } & \multicolumn{2}{c}{ Total } \\
& $\mathbf{N}^{\circ}$ & $\mathbf{0}$ & $\mathbf{N}^{\circ}$ & $\mathbf{0}$ & $\mathbf{N}^{\circ}$ & $\mathbf{\%}$ & $\mathbf{N}^{\circ}$ & $\mathbf{\%}$ \\
\hline 1 & 7 & 17,5 & 33 & 82,5 & - & - & $\mathbf{4 0}$ & $\mathbf{1 0 0}$ \\
2 & 3 & 17,6 & 14 & 82,4 & - & - & $\mathbf{1 7}$ & $\mathbf{1 0 0}$ \\
3 & 2 & 25 & 5 & 62,5 & 1 & 12,5 & $\mathbf{8}$ & $\mathbf{1 0 0}$ \\
4 & 2 & 40 & 3 & 60 & - & - & $\mathbf{5}$ & $\mathbf{1 0 0}$ \\
5 & 1 & 33,3 & 1 & 33,3 & 1 & 33,3 & $\mathbf{3}$ & $\mathbf{1 0 0}$ \\
6 & - & - & 2 & 100 & - & - & $\mathbf{2}$ & $\mathbf{1 0 0}$ \\
\hline Total & $\mathbf{1 5}$ & $\mathbf{2 0}$ & $\mathbf{5 8}$ & $\mathbf{7 7 , 3}$ & $\mathbf{2}$ & $\mathbf{2 , 7}$ & $\mathbf{7 5}$ & $\mathbf{1 0 0}$ \\
\hline
\end{tabular}

Ao analisarmos o número de punções venosas realizadas nos pacientes internados constatamos que $77,3 \%$ foram realizadas pelo auxiliar de enfermagem, $20 \%$ pelo enfermeiro e $2,7 \%$ não identificados (Tabela 1). A categoria, não identificada, relaciona-se com a interrupção da terapia intravenosa ter ocorrido no centro cirúrgico, o que impossibilitou a obtenção destes dados.

A análise deste tópico muito nos inquietou, particularmente no que se refere ao número de punções venosas realizadas pelo enfermeiro. Apreendemos que apenas 10 pacientes se beneficiavam das habilidades deste profissional. No entanto, reconhecemos que é uma das funções esperadas no desempenho do auxiliar de enfermagem. Considerando o conhecimento e habilidade do profissional enfermeiro e o momento crítico que antecede a cirurgia, acreditamos que deveria ser estimulada a participação deste profissional, pois o episódio da cirurgia pode ter um efeito assustador para o paciente.

Os dados obtidos em relação as características da rede venosa para a inserção do dispositivo apontam que $59 \%$ e $16 \%$ destas apresentam-se visíveis e palpáveis e não visíveis e palpáveis, respectivamente. Apenas $25 \%$ apresentam-se não visíveis e não palpáveis. Os resultados encontrados nessa investigação mostram que $75 \%$ das condições da rede venosa do paciente foram consideradas satisfatórias para a realização do procedimento. Entretanto, cabe ressaltar que além das condições supra citadas, existem variáveis externas que podem interferir no sucesso da terapia intravenosa, FULTON (1997). Foram consideradas como variáveis externas: a qualificação profissional, habilidades, idade do paciente, patologia de base e sexo.

Observamos que de um total de 40 pacientes 
internados foram realizados 75 punções venosas e que, destes $57,5 \%$ submeteram-se a uma punção, $22,5 \%$ a duas, $7,5 \%$ a três, $5 \%$ a quatro e $7,5 \%$ a cinco ou mais. Ao analisar o número de punções venosas constatamos que em $42,5 \%$ foram realizadas mais de uma punção. FULTON (1997) afirma que o número de inserções, tipo de dispositivo e tempo de duração constituem fatores de risco para complicações em pacientes submetidos a terapia intravenosa. Assim, os resultados encontrados nesta investigação reforçam a necessidade de atenção permanente da equipe de enfermagem quanto aos critérios de escolha da rede venosa e quanto aos cuidados subseqüentes para a sua manutenção.

Quanto a instalação do dispositivo, os dados apontam que $66,7 \%$ das punções venosas foram realizadas no membro superior esquerdo e $33,3 \%$ no direito. Em relação ao número de punções venosas, $30,7 \%$ foram realizadas no braço dominante. NASCIMENTO (1994) ao considerar o aspecto do lado de maior domínio de movimentos do paciente, observou uma discreta diferença em favor da escolha pelo lado não dominante. Em contrapartida, o nosso estudo aponta que um terço do total das punções foram realizadas no braço dominante.

Ao investigar o tipo de restrição referida encontrada no braço não dominante para instalação do dispositivo, obtivemos que em $48 \%$ relacionam-se com rede venosa de difícil acesso, $17 \%$ descartado local após a avaliação prévia, 13\% não foi finalizada a punção após primeira tentativa, $13 \%$ não foi avaliado, sendo que somente $9 \%$ constitui-se de motivo referido pelo paciente. Em contrapartida, os nossos achados indicam que em $75 \%$ as condições da rede venosa apontaram que as características eram favoráveis à punção. Portanto, os motivos referidos carecem de outras investigações para certificar-se dos reais motivos desta escolha.

Em relação ao local de instalação da terapia intravenosa verificamos que, $48 \%$ das punções venosas realizadas foram na face anterior do antebraço, $21 \%$ no dorso da mão e $15 \%$ na veia mediana da fossa anticubital. Estes achados vêm ao encontro dos dados obtidos por NASCIMENTO (1994) e HECKER (1989). Segundo dados da literatura espera-se que, em ordem decrescente, os locais de instalação da terapia intravenosa sejam as veias do antebraço e plexo metacarpiano. Isto justificase face aos critérios adotados para escolha da rede venosa a saber: calibre, trajeto, elasticidade, palpação, visibilidade e distância das regiões articulares, visando a evitar complicações decorrentes deste procedimento. Cabe ressaltar que em $15 \%$ as punções foram realizadas em veia mediana na fossa anticubital o que poderia dificultar o conforto e a movimentação do paciente. Em relação as veias do plexo metacarpiano, segundo BRANNEN \& SURETTE (1997) a localização do dispositivo apresenta uma alta incidência de provável infecção mais que outros locais.

Ao considerar o tipo de dispositivo adotado para punção venosa, constatamos que $97,3 \%$ eram cateter plástico e 2,7\% escalpe. Esses dados mostram que a opção pelo tipo de dispositivo adotado está de acordo com o tipo de paciente admitido na clínica cirúrgica. Isto nos leva a pensar que a equipe de enfermagem tem se preocupado em avaliar a patologia de base e o porte da cirurgia ao escolher o dispositivo a ser utilizado no procedimento. Assim, concordamos com NASCIMENTO (1994) que a escolha inadequada do dispositivo pode pesar na qualidade da assistência de enfermagem de forma a ocasionar sofrimento adicional e sobretudo desnecessário ao paciente.

Quando observamos o calibre do cateter plástico a ser utilizado na punção venosa constatamos que, 38,4\% eram de calibre $n^{\circ} 20$ e $37 \% n^{\circ} 22$. Em relação ao calibre do scalp obtivemos que um era $n^{0} 21$ e o outro $n^{0} 25$. Constatamos que quando o paciente é submetido a punções venosas subseqüentes, o calibre do dispositivo utilizado é alterado. Esta alteração pode estar associada a escolha inadequada do calibre do dispositivo em relação à condição da rede venosa. Outra justificativa pode estar relacionada a sua condição pós-cirúrgica, onde os critérios estabelecidos para escolha do calibre do dispositivo poderão sofrer modificações face a condição do estado do paciente. Isto reforça a necessidade de estabelecer protocolos na clínica cirúrgica para orientar a conduta em relação ao procedimento de instalação da terapia intravenosa.

Encontramos que $68 \%$ dos profissionais que realizavam a punção venosa não usavam luvas durante o mesmo, e que somente $14,7 \%$ as utilizaram. Segundo o Comitê de Aconselhamento das Práticas de Controle de Infecção Hospitalar as Precauções-Padrão são indicadas para reduzir o risco de transmissão de pátogenos veiculados pelo sangue e daqueles provenientes de outros fluidos corpóreos. Tal fato deve exigir por parte da equipe de saúde o uso de equipamentos de proteção adequados, a fim de evitar infecções hospitalares e possíveis acidentes ocupacionais. Frente aos achados, deixamos registrada a necessidade emergente de revisão das práticas de precauções-padrão sobre a utilização de luvas durante a realização da punção venosa, bem como, os procedimentos relativos a anti-sepsia da pele do paciente.

Em relação a anti-sepsia da pele constatamos que $66,7 \%$ dos funcionários a realizam com algodão e álcool, $70 \%$ técnica adequada, 16\% com algodão e álcool, $70 \%$ técnica inadequada e $17,3 \%$ não identificado. Conceituamos como técnica inadequada, quando o funcionário teve as seguintes condutas: garroteamento prolongado, bater na veia e colocar o dedo sobre o local da inserção após a anti-sepsia, durante o preparo do local e a inserção do dispositivo. 


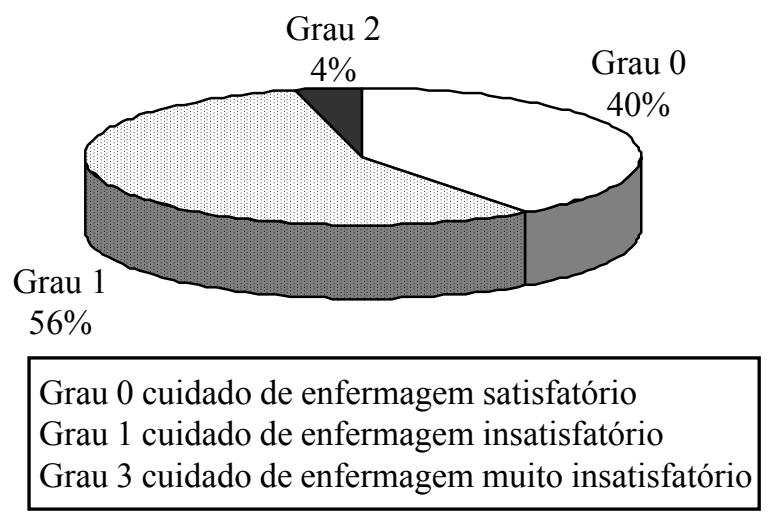

Figura 1 - Distribuição da freqüência das punções venosas realizadas em pacientes internados em um hospital de grande porte, segundo o grau de cuidado de enfermagem, Ribeirão Preto - SP, 1999

Ao analisarmos o cuidado de enfermagem implementado durante o período em que o paciente encontra-se em terapia intravenosa, constatamos que 56\% e $4 \%$ referem-se ao grau 1 e 2 , respectivamente, perfazendo um total de 60\% (Figura 1). Segundo LUNDGREN et al. (1993) o grau de cuidado de enfermagem pode ser alterado com o tempo de permanência do dispositivo in situ, sendo que, após o segundo dia de instalação há comprometimento da qualidade deste cuidado. Este comprometimento pode estar associado a falta de observação de sinais e sintomas de possíveis complicações por parte da equipe de enfermagem. Os nossos dados apontam que $60 \%$ do cuidado de enfermagem em relação a punção venosa foi classificado como insatisfatório e muito insatisfatório. Esta situação poderia ser minimizada através de padronização de condutas em relação a terapia intravenosa, diante de medidas tais como: observação sistemática, documentação no prontuário do paciente e comunicação da equipe durante a passagem de plantão. Acreditamos que a qualidade do cuidado de enfermagem para este procedimento deverá ser estimulada, cabendo aos enfermeiros assumir esta responsabilidade.

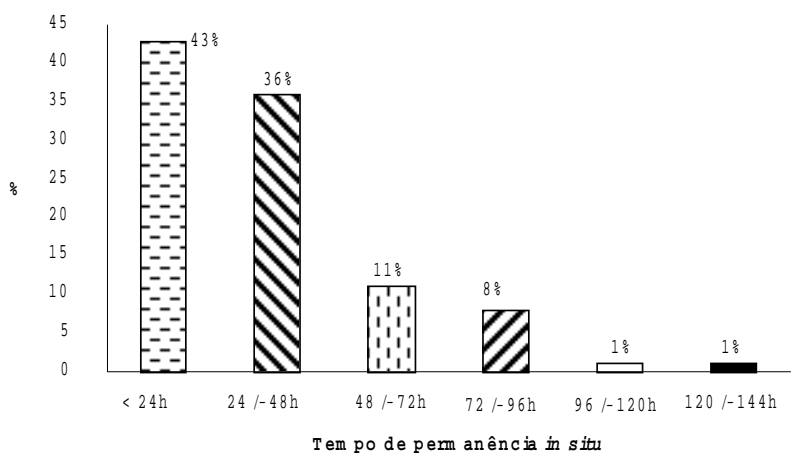

Figura 2 - Distribuição da freqüência de pacientes internados em um hospital de grande porte, segundo o tempo de permanência do dispositivo venoso periférico in situ, Ribeirão Preto - SP, 1999
Ao analisar o tempo de permanência do dispositivo venoso periférico in situ, verificamos que $43 \%$ dos pacientes permaneceram por um período de tempo menor que 24 horas, $36 \%$ de 24 a 48 horas e $11 \%$ de 48 a 72 horas e que, apenas 10\% mais que 72 horas (Figura 2). A literatura sobre o tempo adequado de permanência do dispositivo venoso periférico in situ tem tido controvérsias, variando de 48 a 72 horas LUNDGREN et al. (1993); NASCIMENTO (1994); BRANNEN \& SURETTE (1997); FULTON (1997) e BREGENZER et al. (1998). Segundo LUNDGREN et al.(1993), os aspectos que o enfermeiro deve estar atento visando melhorar a qualidade do cuidado de enfermagem prestado estão relacionados ao tempo de permanência in situ e boa fixação para evitar complicações. Em nosso estudo, verificamos que em $47 \%$ dos pacientes o tempo de permanência variou de 24 a 72 horas, sendo que, em apenas $10 \%$ o tempo de permanência foi maior que 72 horas. Constatamos que os resultados obtidos por nós em relação ao tempo de permanência está de acordo com o preconizado na literatura. No entanto, ao analisar estes dados em conjunto com o grau do cuidado de enfermagem, isto torna-se preocupante. Esta preocupação pauta-se considerando os resultados apontados por MAKI \& RINGER (1991) os quais referem que o tempo de permanência in situ por mais de 48 horas aumenta aproximadamente $30 \%$ o risco de flebite e que a partir de 72 horas o risco eleva-se para 39 a 49\%. Isto impõe a equipe de enfermagem estabelecer critérios rígidos e o desenvolvimento de pesquisas, a fim de buscar subsídios que possam dar sustentação as ações de enfermagem em relação a terapia intravenosa.

Ao considerar o motivo de interrupção da terapia intravenosa, os dados apontam que 52\% relacionam-se término indicação da terapia intravenosa, $20 \%$ infiltração, $8 \%$ transfixação da veia, $5 \%$ obstrução do dispositivo e $5 \%$ infiltração e hematoma, 3\% flebite e término de terapia, $3 \%$ dor relatada pelo paciente e $4 \%$ outros motivos. Dentre os motivos de interrupção destaca-se a infiltração com $20 \%$, corroborando o grau 1 e 2 do cuidado de enfermagem encontrados no presente estudo. Dessa forma, medidas são necessárias para evitar a infiltração, porque sua ocorrência é motivo para interrupção da terapia intravenosa e conseqüente reinstalação. Portanto, a sua ocorrência freqüente resultará na inacessibilidade de veias periféricas, tornando necessário um acesso via central, expondo o paciente a riscos. Os resultados de NASCIMENTO (1994) mostram que de 165 motivos de interrupção 34 foram devido a ocorrência de flebite. Em contrapartida, nossos dados mostram que a ocorrência da flebite não foi considerado uma complicação para a interrupção da terapia intravenosa. Quando associada a outros motivos, tais como: flebite e obstrução do dispositivo e flebite e 
término da terapia, a flebite pôde ser considerada como um motivo de interrupção, correspondendo a 3 motivos de um total de 75. Esses dados podem ser comparados com os resultados obtidos por LUNDGREN et al. (1993), onde os primeiros sinais e sintomas de infecção relacionados ao dispositivo venoso periférico podem ocorrer no segundo dia de terapia intravenosa, porém o dispositivo não é retirado. Isto refletiu a necessidade do treinamento em serviço relacionado a este procedimento.

\section{CONCLUSÕES}

A presente investigação permitiu-nos concluir que: o paciente cirúrgico deste estudo constitui-se em sua maioria de cirurgia geral $(60 \%)$, entre 48 a 78 anos $(50 \%)$ e a patologia de base está relacionada a hérnias incisionais e ingüinais $(37,5 \%), 77,3 \%$ das punções intravenosas foram realizadas pelo auxiliar de enfermagem, as características da rede venosa em $75 \%$ apresentaram condições favoráveis; $57,5 \%$ dos pacientes submeteram-se a uma única punção; a maioria $(66,7 \%)$ da instalação foi realizada no membro superior esquerdo; no braço não dominante $(69,3 \%)$; o tipo de restrição referida para instalação no braço não dominante em $48 \%$ refere-se a rede venosa de difícil acesso; $48 \%$ o local de instalação foi na face anterior do antebraço; $97,3 \%$ o tipo de dispositivo eleito foi o cateter plástico; $38,4 \%$ o calibre do cateter plástico foi $\mathrm{n}^{\circ} 20$. A maioria dos profissionais da equipe de enfermagem (68\%) não utilizaram luvas durante o procedimento. Em relação ao grau do cuidado de enfermagem $60 \%$ refere-se ao grau 1 cuidado de enfermagem insatisfatório e grau 2 cuidado de enfermagem muito insatisfatório. O tempo de permanência do dispositivo in situ foi de 24 a 72 horas em $47 \%$ dos pacientes. Quanto ao motivo de interrupção e complicações da terapia intravenosa $20 \%$ relacionaramse a infiltração; $8 \%$ transfixação da veia; 5,4\% infiltração e hematoma; 5,3\% obstrução do dispositivo. Acreditamos que, os dados encontrados nesta investigação merecem ser analisados pelos profissionais da equipe de enfermagem com vistas ao aprimoramento de programas de educação em serviço relacionado a terapia intravenosa.

\section{COMPLICATIONS DUE TO INTRAVENOUS THERAPY IN SURGICAL PATIENTS}

Nursing plays an important role in prevention and reduction of complications related to venous access. This study had the purpose to find complications derived from intravenous therapy. Major data were: $77.3 \%$ of the venous accesses were performed by auxiliary nursing; $68 \%$ of the procedures were performed without gloves; $60 \%$ of the accesses were not satisfactorily performed by nurses; $47 \%$ of the cannulae were "in situ" between 24 and 72 hours; among the complications, $20 \%$ were related to infiltration, $5.4 \%$ to infiltration and hematoma and $5.3 \%$ to obstruction. Data suggest the need to improve the education of the nursing team about intravenous therapy.

KEY WORDS: venous infusions/complications, nursing care

\section{COMPLICACIONES CAUSADAS POR LA TERAPIA INTRAVENOSA EN PACIENTES QUIRÚRGICOS}

Enfermería desempeña un papel primordial en la prevención y reducción de complicaciones relacionadas con el acceso venoso. El estudio tiene como objetivo la identificación de complicaciones ocurridas en la terapia intravenosa. Los datos más relevantes que fueron obtenidos son: 77,3\% de las punciones fueron realizadas por auxiliares de enfermeria; $68 \%$ no usaron guantes durante el procedimiento; $60 \%$ de las punciones tuvieron cuidado de enfermería insatisfactorio; 20\% relacionados con infiltraciones; 5,4\% con infiltraciones y hematomas y, 5,3\% con obstrucciones. Los datos sugieren la necesidad de capacitar al equipo de enfermería con respecto a la terapia intravenosa.

TÉRMINOS CLAVES: infusiones venosas/complicaciones, cuidado de enfermería

\section{REFERÊNCIAS BIBLIOGRÁFICAS}

01. BRANNEN, S.; SURETTE, D. Monitoring IV-site infections. Can. Nurse, v. 93 ,n. 3, p. 49-50, 1997.

02. BREGENZER, T.; CONEN, D.; SAKMANN, P.; WIDMER, A.F. Is routine replacement of peripheral intravenous catheters necessary? Arch. Intern Med., v. 158, n. 2, p. 151-6, 1998.
03. FULTON, J.S. Long-term vascular access devices. Annu. Rev. Nurs. Res., v. 15, p. 237-62, 1997. Chapter 10.

04. HECKER, J.F. Failure of intravenous infusions from extravasation and phlebitis. Anesth. Intensive Care, v. 17, n. 4, p. 433-9, 1989. 
05. LUNDGREN, A.; JORFELDT, L.; EK, A.C. The care and handling of peripheral intravenous cannulae on 60 surgery and internal medicice patients: an observation study. J. Adv. Nurs.,v. 6, n. 18, p. 963-71, 1993.

06. MAKI, D.G.; RINGER, M. Risk factors for infusionrelated phlebitis with small peripheral venous catheters. Ann. Intern Med., v. 114, n. 10, p. 845-54, 1991.

07. NASCIMENTO, E. Infusão intravenosa em veia periférica: associações entre motivos de interrupção e fatores envolvidos. São Paulo, 1994. Dissertação (Mestrado) - Escola Paulista de Medicina.
08. NORWOOD, S.; RUBY, A.; CIVETTA, J.; CORTES, V. Catheter-related infections and associated septicemia. Chest, v. 99, n. 4, p. 968-75, April 1991.

09. POLIT, D.F.; HUNGLER, B.P. Fundamentos de pesquisa em Enfermagem. Porto Alegre: Artes Médicas, 1995.

10. VIEIRA, M.R.R. Avaliação da qualidade do cuidado de enfermagem na infusão endovenosa periférica contínua prestado ao pré-escolar. São José do Rio Preto, 1998. Dissertação (Mestrado) - Faculdade de Medicina de São José do Rio Preto. 